\title{
A System-Level Overview of Near-Field Meta-Steering
}

\author{
Muhammad U. Afzal ${ }^{(1)}$, Karu P. Esselle ${ }^{(1)}$, and Ali Lalbakhsh ${ }^{(2)}$ \\ (1) University of Technology Sydney (UTS) \\ Sydney, NSW 2007, Australia \\ (2) Macquarie University, Sydney, NSW 2109, Australia
}

\begin{abstract}
The paper provides a system-level overview of Near-Field Meta-Steering (NFMS) technology. The NFMS is upcoming antenna beam-steering method that uses the physical rotation of pair of thin metasurfaces that are placed in very close proximity to a high-gain feeding base antenna. This method neither uses any active radio frequency (RF) components nor physical tilting of any antenna part. It is for these reasons that this method yield antenna systems that superior to traditional electronically scanned phased array and mechanically rotated beamsteering antennas. The antenna systems can be developed for a range of applications including inflight connectivity, low-cost satellite terminal antennas to provide connectivity at remote places, and high-power micro- and millimeterwave applications. The dynamic phase transformation that is achieved by the rotation of two metasurfaces, in a proofof-concept prototype reported in 2017, indicate that an antenna beam can be scanned in a conical region having an apex angle of $102^{\circ}$.
\end{abstract}

\section{Introduction}

The Near-Field Meta-Steering (NFMS) is an emerging high-gain antenna beam-steering technique that combines the unique and attractive features of traditional electronic and mechanical steering antennas. The technique is inspired from an optical beam-scanning concept that is implemented using pair Risley prisms [1,2]. The optical scanning led to the development of beam-scanning antennas using dielectric wedges [3,4] and later using phase-shifting surfaces [5]. The major breakthrough was the use of near-field metasurfaces to steer the beam of medium-to-high gain antennas, which was first reported in 2017 [6].

The electromagnetic (EM) part of the NFMS based antenna system comprises a high-gain antenna, which is also referred to as base antenna, and a pair of phase-gradient metasurfaces (PGMs) to introduce a specific phase shift in the field radiated by the base antenna [6]. The two PGMs are placed above the base antenna, in the near-field region, and at not more than one wavelength spacing apart. The high-gain base antenna is totally stationary and the PGMs are physically rotated to scan the antenna beam in an angular region.

The NFMS yields antenna systems that are better than contemporaries in many ways. These antenna systems have extremely low height profile - the height of antenna system reported in [6] is only $1.3 \lambda_{0}$, where $\lambda_{0}$ is the free-space wavelength at the operating frequency. The actual radiator in the antenna system that has radio frequency (RF) connection, i.e. base antenna, is stationary and hence do not require any RF rotary joints used in other mechanical steering systems. The electrical power is only needed for the low-power motors that are used to rotate the pair of PGMs. In contrast, electronically scanned phased arrays need at least one phase shifter for each antenna element in the array. The NFMS antenna systems can be developed for high-power applications using specially designed all-metal type metasurfaces $[7,8]$. During the scan, the antenna system maintains planar profile unlike physically rotated reflector dishes that need $3 \mathrm{D}$ volume to physically move the structure.

The NFMS has inspired others to extend the concept in various forms. An all-dielectric version of beam-steering system was investigated through numerical simulations using graded-dielectric plates (GDPs) [9]. In another following work, dual-polarized beam-steering antenna systems were investigated [10]. A more accurate phase method was reported to predict direction of antenna beam using rotation angles of the PGMs [11]. A more recent investigation have reported optimized pair of PGMs to reduce undesirable grating lobes [12].

Considering the interest of the research community, this paper aims at introducing system level overview of the NFMS based antenna systems without focusing on detailed design procedures of individual components. The paper is organized such that first system description is given in Section II. The propagation of field from two PGMs will be explained in Section III and finally paper is concluded at the end.

\section{System Level Description}

A pictorial view of the classic NFMS based antenna is shown in Fig. 1. A planar base antenna is depicted in the figure but any other high-gain antenna that has a large radiating aperture can be used as the base antenna [13-16]. The base antenna typically has a beam fixed in the broadside direction. The two PGMs (M1 and M2) are placed above the base antenna and are individually designed to tilt the antenna beam away from the broadside in a specific direction. It is for this reason that these metasurfaces are sometime also referred to as beam-tilting 


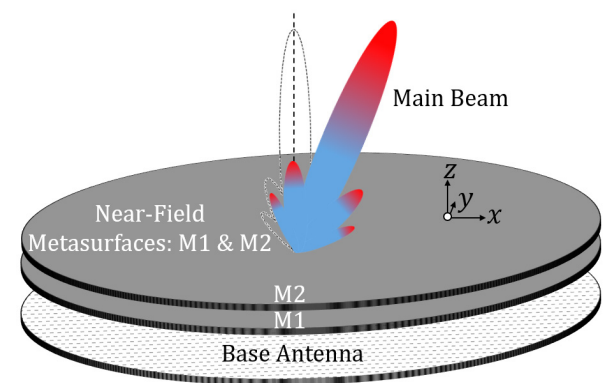

Fig. 1: Perspective view of a Near-Field Meta-Steering base antenna system. The two metasurfaces M1 and M2 are physically rotated around the antenna axis (z-axis here).

metasurfaces. In this classical configuration, the M2 is independent of the choice of base antenna. However, the M1 varies with a base antenna.

If the base antenna has nearly uniform aperture phase distribution in the near-field region, then M1 and M2 are identical. On the other hand, if the base antenna has nonuniform aperture phase distribution in the near-field region then M1 is modified to cater for the phase non-uniformity. In some cases, as done in [9], an additional layer of metasurface can be introduced before M1 and M2 to first remove the non-uniformity in aperture phase distribution $[13,14]$. In this latter case, M1 and M2 can be identical but this comes at the cost of an additional surface.

The fundamental element of a PGM is a spatially distributed phase-shifting unit-cell elements. The unit-cell elements locally implement the phase profile of a PGM. The operating principle of the PGMs is same as that of phased arrays. A typical PGM has unit cells having lateral dimensions smaller than $\lambda_{0} / 2[6,12]$. In some all-metal type PGMs unit-cell elements slightly larger than $\lambda_{0} / 2$ are also used. The detailed discerption of unit-cell elements in phase transforming metasurfaces articles including planar lenses and will be discussed here.

A PGM introduces progressive phase shift, in the electric field propagating through it, using spatially distributed unit-cell elements. The unit-cells are arranged such that phase shift increases with a constant step along one of the linear axes of the metasurface and is constant along the orthogonal axis. The step size or $\Delta \Phi$ is calculated following antenna array theory and depends on the required tilting angle, the lateral size of the unit-cell element, and the operating frequency of the antenna system [6]. A typical phase profile of the PGM along the increasing phase axis is shown in Fig. 3. The ideal phase of a PGM is shown with a thick black line in the figure. Since the phase repeats after a cycle of $360^{\circ}$, this phase can be wrapped between 0 and $360^{\circ}$ across the metasurface aperture. The number of periodic cycles of phase progression depends on the aperture size of the metasurface. As an example, an arbitrary sized (D) PGM is drawn with grey color indicating the phase progression and wrapped phase cycles across the aperture of the PGM in Fig. 2(b). In this figure uniform color along vertical axis indicate placement of same phase-shifting unit cells and hence constant phase shift. The varying color along horizontal axis indicate different phase-shifting unit cells having progressive phase

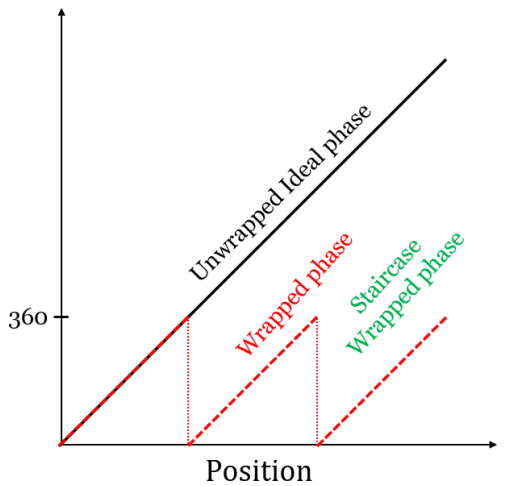

(a)

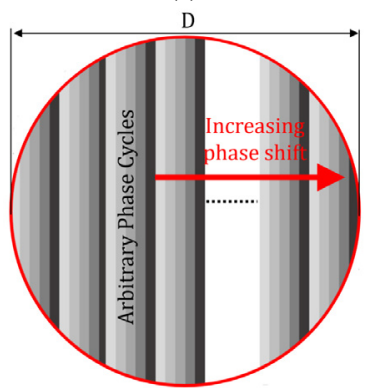

(b)

Fig. 2: (a). Transmission phase profile of a phase-gradient metasurface (PGM), (b). An arbitrary circular PGMs with wrapped phase profile depicted with grey color.

shift along the horizontal axis. The linear axis along which the phase shift increases is important because the metasurface tilts the antenna beam along this axis.

\section{Field Propagation Through Pair of Parallel Phase-Gradient Metasurfaces (PGMs)}

The classic configuration of NFMS system, shown in Fig. 1 having a base antenna and an identical pair of metasurfaces is explained here. It is assumed that the antenna has uniform aperture phase distribution and the electric field at the input of first metasurface (M1) has planar phase front. The M1 introduces a linearly increasing phase shift as pictorially shown in Fig. 4, which effectively tilts the beam at an offset angle.

The field propagation through second metasurface (M2) can be explained for two extreme cases separately: (a) when M1 and M2 are aligned (b) when M1 and M2 are pointing in opposite directions. These two cases are pictorially depicted in Fig. 4. In Fig. 4(a), when the

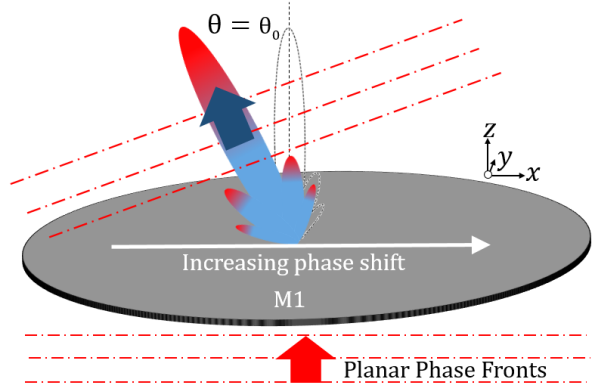

Fig. 3: Functional depiction of the first of two metasurface used in the steering system. 


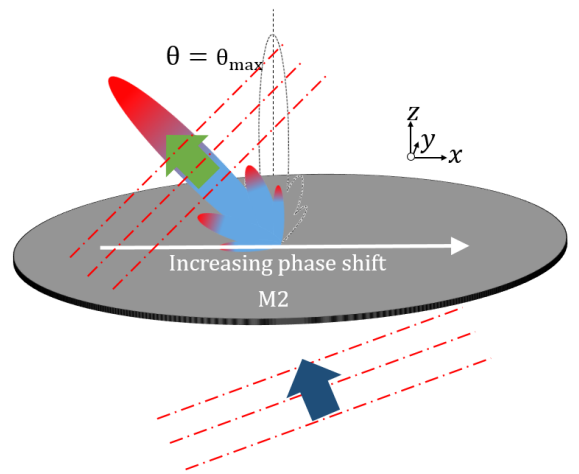

(a)

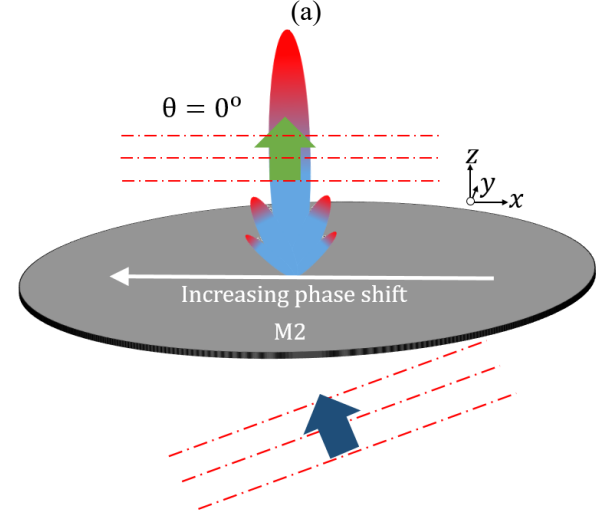

b.

Fig. 4: Field propagation through second metasurface (a). When axis of increasing phase shift of the M2 is aligned with M1 (b). When axis of increasing phase shfit of M2 is in opposite direction to that of M1.

increasing phase shift of the two metasurfaces are aligned $(+\mathrm{x}$-axis here), the slope of electric field phase at the output of M2 is increased further and effectively, the beam tilt angle reaches maximum elevation angle. On the other hand, when phase progression axes of M2 (towards $-\mathrm{x}-$ axis) is in opposite direction to that of M1 (+x-axis in Fig. $3)$, the slope of phase front at the output of M2 reduces to zero and beam moves to the broadside direction. Using this system of parallel metasurfaces, the slope of the electricfield phase at the output of M2 can be adjusted between two extreme cases. This gives direct control to change antenna beam direction between broadside direction and maximum elevation angle. Further details on beamscanning and metasurfaces rotation can be found in any of earlier publications $[6,9,12]$.

One of the challenges associated with the phase wrapping and periodic nature of a PGM (as depicted in Fig. 2(b)) is that it leads to grating type side lobes. The issues of grating lobes can be addressed to some extent using an optimized pair of metasurfaces in [12] that have steeper phase gradients. It is concluded that a steeper phase-gradient leads to smaller number of grating lobes and high directivity in the main beam direction.

\section{Conclusion}

The paper presents a basic system-level overview to understand the working of Near-Field Meta-Steering technology (NFMS). The key to achieving beam-steering is to dynamically control the aperture phase distribution by rotating the two phase-gradient metasurfaces (PGMs). The recent research interest from several groups across the world highlights the immense importance and unique characteristics of this beam-steering technology. The full potential of NFMS can be utilized to develop connectivity solution for several upcoming applications including mobile-satellite connectivity.

\section{References}

[1] M. Ostaszewski et al., "Risley prism beam pointer," 2006, vol. 6304 , pp. 630406-630406-10.

[2] Q. Peng, X. Wang, G. Ren, H. Chen, L. Cao, and J. Wang, "Analytical direct solutions of the Risley prism systems for tracking and pointing," Appl. Opt., vol. 53, no. 13, pp. C83-C90, 2014/05/01 2014, doi: 10.1364/AO.53.000C83.

[3] C. D. McEwen and M. R. Khan, "Beam Steering Method with Improved Sidelobe Response using Dielectric Wedges for Satellite TV Reception," in Microwave Conference, 1984. 14th European, 1984, pp. 681-685.

[4] B. J. Tame and N. A. Stutzke, "Steerable Risley Prism antennas with low side lobes in the Ka band," in Wireless Information Technology and Systems (ICWITS), 2010 IEEE International Conference on, Aug. 28 2010-Sept. 32010 2010, pp. 1-4, doi: 10.1109/ICWITS.2010.5611931.

[5] N. Gagnon and A. Petosa, "Using Rotatable Planar Phase Shifting Surfaces to Steer a High-Gain Beam," IEEE Trans. Antennas Propagat., vol. PP, pp. 1-1, 2013.

[6] M. U. Afzal and K. P. Esselle, "Steering the Beam of Medium-toHigh Gain Antennas Using Near-Field Phase Transformation," IEEE Transactions on Antennas and Propagation, vol. 65, pp. 1680-1690, 2017.

[7] X. Zhao, C. Yuan, L. Liu, S. Peng, Q. Zhang, L. Yu, et al., "AllMetal Beam Steering Lens Antenna for High Power Microwave Applications," IEEE Transactions on Antennas and Propagation, vol. 65, pp. 7340-7344, 2017.

[8] X. Zhao, C. Yuan, L. Liu, S. Peng, Q. Zhang, and H. Zhou, "AllMetal Transmit-Array for Circular Polarization Design Using Rotated Cross-Slot Elements for High-Power Microwave Applications," IEEE Transactions on Antennas and Propagation, vol. 65 , pp. 3253-3256, 2017.

[9] M. U. Afzal, A. Lalbakhsh and K. P. Esselle, "Electromagnetic-wave beam-scanning antenna using near-field rotatable graded-dielectric plates", Journal of Applied Physics, vol. 124, no. 23, pp. 234901$234912,2017$.

[10] T. Lou, X. Yang, H. Qiu, Z. Yin, and S. Gao, "Compact DualPolarized Continuous Transverse Stub Array With 2-D Beam Scanning," IEEE Transactions on Antennas and Propagation, vol. 67, pp. 3000-3010, 2019.

[11] J. Wang and Y. Ramhat-Samii, "Phase Method: A More Precise Beam Steering Model For Phase-Delay Metasurface based Risley Antenna," in 2019 URSI International Symposium on Electromagnetic Theory (EMTS), 2019, pp. 1-4.

[12] K. Singh, M. U. Afzal, M. Kovaleva, and K. P. Esselle, "Controlling the Most Significant Grating Lobes in Two-Dimensional BeamSteering Systems with Phase-Gradient Metasurfaces," IEEE Transactions on Antennas and Propagation, pp. 1-1, 2019.

[13] G. Minatti et al., "Modulated Metasurface Antennas for Space: Synthesis, Analysis and Realizations," in IEEE Transactions on Antennas and Propagation, vol. 63, no. 4, pp. 1288-1300, April 2015.

[14] M. Ando, K. Sakurai, N. Goto, K. Arimura and Y. Ito, "A radial line slot antenna for $12 \mathrm{GHz}$ satellite TV reception," in IEEE Transactions on Antennas and Propagation, vol. 33, no. 12, pp. 13471353, December 1985.

[15] N. Y. Koli, M. U. Afzal, K. P. Esselle and M. Zahidul Islam, "A Radial Line Slot Array Antenna with Improved Radiation Patterns for Satellite Communication," 2019 13th European Conference on Antennas and Propagation (EuCAP), Krakow, Poland, 2019, pp. 12 .

[16] N. Y. Koli, M. U. Afzal, K. P. Esselle and M. Z. Islam, "Investigation on Aperture Field Distribution of Circularly Polarised Radial Line 
Slot Array Antennas," 2018 IEEE Asia-Pacific Conference on Antennas and Propagation (APCAP), Auckland, 2018, pp. 462-463.

[17] M. U. Afzal and K. P. Esselle, "A low-profile printed planar phase correcting surface to improve directive radiation characteristics of electromagnetic band gap resonator antennas," IEEE Transactions on Antennas and Propagation, vol. 64, pp. 276-280, 1/1 2016.

[18] A. Lalbakhsh, M. U. Afzal, and K. P. Esselle, "Multiobjective particle swarm optimization to design a time-delay equalizer metasurface for an electromagnetic band-gap resonator antenna," IEEE Antennas and Wireless Propagation Letters, vol. 16, pp. 912$915,2017$. 